\title{
Assessment of Potential Targets for Deep Brain Stimulation in Patients With Alzheimer's Disease
}

\author{
Mayur Sharma $^{\text {a }}$, Milind Deogaonkar ${ }^{\mathrm{a}, \mathrm{b}}$, Ali Rezai ${ }^{\mathrm{a}}$
}

\begin{abstract}
Alzheimer's disease (AD) is a progressive neurodegenerative disorder affecting 36 million people worldwide and 5.2 million in the United States. The pathogenesis of AD is still elusive. Accumulations of abnormal proteins (beta amyloid and tau protein), inflammatory cascades, abnormal responses to oxidative stress and alteration in oxidative metabolism have been implicated in AD. There are few effective therapeutic options available for this disorder at present. Neuromodulation offers a novel treatment modality for patients with AD. The databases of Medline and PubMed were searched for various studies in English literature describing the deep brain stimulation (DBS) in patients with AD. Various animal and human clinical studies have shown promising initial results with bilateral DBS targeting various anatomical nodes. In this review, we attempt to highlight the pathophysiology, neural circuitry and potential neuromodulation options in patients with AD. In appropriately selected patients, DBS can potentially delay the cognitive decline, enhance memory functions and can improve the overall quality of life. However, further randomized controlled trials are required to validate the efficacy of neuromodulation and to determine the most optimal target for $\mathrm{AD}$.
\end{abstract}

Keywords: Deep brain stimulation; Neuromodulation; Alzheimer's disease

\section{Introduction}

Alzheimer's disease (AD) is a progressive neurodegenerative disorder. This disorder was first described by Dr. Alois Alzheimer in 1901 in a 51-year-old woman with progressive memory loss [1]. AD is a leading cause of cognitive disability and

Manuscript accepted for publication April 13, 2015

${ }^{a}$ Center of Neuromodulation, Wexner Medical Center, The Ohio State University, Columbus, OH 43210, USA

${ }^{\mathrm{b} C}$ Corresponding Author: Milind Deogaonkar, Department of Neurosurgery, Center of Neuromodulation, Wexner Medical Center, The Ohio State University, 480 Medical Center Drive, Columbus, OH 43210, USA.

Email: Milind.Deogaonkar@osumc.edu

doi: http://dx.doi.org/10.14740/jocmr2127w dementia worldwide and in the United States. The number of people affected by $\mathrm{AD}$ has increased from 26.6 million in 2006 to 36 million in 2014 worldwide [2, 3] (http://www.alzheimers. net/resources/alzheimers-statistics/). Of these 36 million AD patients, 5.2 million are in the United States, of which 200,000 patients are less than 65 years of age (young onset AD). A new patient is diagnosed with $\mathrm{AD}$ in the United States every 67 s (http://www.alz.org/alzheimers_disease_facts_and_figures. asp\#prevalence). In 2014, the healthcare expenditure on AD has been estimated to be 605 billion US dollars worldwide and 150 billion US dollars in the United States [3]. This enormous burden of $\mathrm{AD}$ poses significant challenges to the health care providers and the caregivers.

The current management options for $\mathrm{AD}$ include medications aimed at potentiating the cholinergic pathways (acetyl cholinesterase inhibitors) and mitigating the glutaminergic pathways (N-methyl D-aspartate receptors blockers) [4-6]. Anti-oxidant such as vitamin $\mathrm{E}$ has also been used as medical therapy for AD. These medications provide modest symptomatic benefits in patients with $\mathrm{AD}$, without altering the course of disease [6]. The lack of efficacy and adverse effects of current medications led to exploration of alternative treatment modalities for AD.

Following the success of deep brain stimulation (DBS) surgery in a variety of movement and psychiatric disorders [7], this treatment modality has been explored as a potential therapeutic option in patients with $\mathrm{AD}[5,8]$. In this review, we attempt to highlight the pathophysiology, neural circuitry and potential neuromodulation options in patients with $\mathrm{AD}$.

\section{Pathophysiology and Neural Substrate of AD}

The etiopathogenesis of $\mathrm{AD}$ is not completely elucidated. Based on animal and human studies, a variety of pathogenic mechanisms such as accumulations of abnormal proteins (beta amyloid and tau protein), inflammatory cascades, abnormal responses to oxidative stress and alteration in oxidative metabolism have been implicated in $\mathrm{AD}[2,3,5,9]$. At molecular level, accumulation of beta amyloid protein and tau protein in different regions of the brain has been implicated in the loss of synaptic functions, defective metabolism, impaired cellular repair, cell death and thus development of AD. Hyperphosphorylation of tau protein (microtubule associated protein) leads to 
the formation of microtubule or neurofibrillary tangles which impair the cellular tubular transport mechanism and thus leads to neuronal death [5]. Accumulation of beta amyloid plaques has also been implicated in the pathogenesis of AD. Amyloid precursor protein (APP) with genetic loci on chromosome 21 binds to death receptor 6 and initiates the apoptotic pathways resulting in neuronal loss $[5,9,10]$. This hypothesis supports an earlier onset of AD in patients with trisomy 21. Accumulation of other proteins such as presenilin 1, presenilin 2 and apolipoprotein E4/E4 with genetic loci on chromosomes 14, 1 and 9 respectively has also been implicated in the pathogenesis of $\operatorname{AD}[3,10]$.

These molecular alterations lead to neuronal loss and cerebral atrophy in the different regions of the brain involving frontal, temporal, parietal, hippocampus and entorhinal cortex (EC) $[3,5]$. In addition to localized cellular loss, AD also disrupts the neuronal connections between the cortical and subcortical areas [8]. These neuronal connections are referred as default mode networks (DMN) and functional alterations in these networks have been linked to the memory deficits in patients with $\mathrm{AD}[8,11]$. Thus $\mathrm{AD}$ can be visualized as a systemic disorder affecting several neuronal networks instead of a localized neurodegenerative disorder. In a recent study, approximately one-third of AD cases worldwide have been attributed to potentially modifiable risk factors such as improved education status, vascular risk factors and depression [12].

\section{Literature Search}

The databases of Medline and PubMed were searched for various studies in English literature describing the DBS in patients with AD. All related studies published up to December 2014 were included in this review.

\section{Animal Models of AD}

Various rodent models have been used to demonstrate the efficacy of DBS in modulating the memory circuits and enhancing the neurogenesis $[13,14]$. Hamani et al [14] showed that DBS of the anterior thalamic region in corticosterone-treated rats improved performance and enhanced hippocampal neurogenesis 1 month following stimulation. They postulated that long-term plastic changes and newly formed mature dentate gyrus cells are implicated in such positive findings [14]. Bilateral DBS of the anterior thalamic nucleus has been shown to reversibly increase glucose uptake in the thalamus/hippocampus and decrease uptake in the cingulate and frontal cortex in non-epileptic rats [15]. Decreased glucose uptake in thalamus/ hippocampal region was observed following bilateral lesioning of anterior thalamic nuclei. Another animal study using TgCRND8 mice demonstrated that DBS (high frequency) of the midline thalamic region enhanced short-term memory in the CA1 region of hippocampus and also increased the nonamyloidogenic $\alpha$-secretase activity [13]. These promising results instigated researchers for the clinical trials of DBS for modulating the memory circuits. At present there are six clin- ical trials of DBS in patients with $\mathrm{AD}$ including one at our center [3].

\section{Current DBS Targets Used in Patients With AD}

DBS is a well-established therapy for a variety of medical refractory movement disorders (Parkinson disease (PD), essential tremors and dystonia) with over 100,000 implants worldwide [7]. DBS was first approved by Food and Drug Administration (FDA) for essential tremors in 1997 [16, 17]. Following the success of DBS in refractory movement disorders with more than 20 years of safety records, this modality has been investigated for psychiatric disorders (obsessive-compulsive disorders, major depression, eating disorders, and addiction), traumatic brain injury, post-traumatic stress disorder, Tourette's syndrome, epilepsy, cluster headache, post-stroke pain, amputation pain, neuropathic pain, multiple sclerosis tremor, and impaired conscious state including dementias with varied success [18-28].

\section{Hypothalamic/fornix}

Based on promising results in animal models, various targets have been explored as potential DBS targets to modulate and enhance the memory functions in patients with AD. Hamani et al [29] reported unexpected improvement in autobiographical memories in a patient following bilateral hypothalamic DBS for morbid obesity. DBS increased recollection indicating potentiation of memory circuits involving hippocampus and mesial temporal lobe (limbic circuits) [29]. Based on this initial report, Laxton et al [28] first conducted the phase 1 trial of DBS of hypothalamus/fornix in patients with AD. In this study, six patients with mild AD underwent bilateral chronic constant stimulation of hypothalamus and the anterior border of vertical portion of fornix for 12 months. Monopolar stimulation was applied at $130 \mathrm{~Hz}, 90 \mu$ s pulse width and increasing the voltage by $1.0 \mathrm{~V}$ every $30 \mathrm{~s}$ until side effect is observed or a maximum intensity of $10 \mathrm{~V}(3-3.5 \mathrm{~V})$. Based on AD assessment scale cognitive subscale and the mini-mental state examination (MMSE) scale, two of six patients showed slower rate of cognitive decline and one patient showed improvement in cognitive functions at 6 and 12 months. PET-CT scans following 12 months of continuous stimulation also demonstrated reversal of impaired glucose metabolism in the temporal and parietal lobes in this study [28]. No serious adverse events were noted in this study. Improved glucose metabolism in the cortical and hippocampal circuits following bilateral DBS of fornix at 1 year using PET-CT scans has been demonstrated in five patients with mild AD in an open label study [30]. Increased glucose metabolism was observed in frontal-temporal-parietal-striatalthalamic circuit and frontal-temporal-parietal-occipital-hippocampal circuit which correlated with improvement in global cognitive functions and quality of life [30]. Fontaine et al [31] demonstrated stabilization of memory scores and increased metabolism in the mesiotemporal lobe following bilateral DBS of fornix in a patient with mild to moderate AD at 52 months. 
Bilateral low frequency stimulation of fornix using in-depth electrodes has been shown to improve MMSE in 11 patients with intractable epilepsy over a period of $4 \mathrm{~h}$ [32]. A major caveat is that these are non-randomized studies without placebo control and therefore results should be interpreted with caution.

\section{Nucleus basalis of Meyernet (NBM)}

Based on animal studies [33], NBM has been explored as a potential target for DBS in patients with dementia [34]. Freund et al [34] implanted bilateral NBM DBS in addition to subthalamic nucleus (STN) DBS in a 71-year-old patient with PD-related dementia. Improvement in attention, alertness, drive, concentration and spontaneity was observed following bilateral NBM stimulation. This improvement in cognitive and behavior functions can be attributed to the potentiation of residual cholinergic projections in the memory circuits [34].

\section{EC/hippocampus}

Since EC/hippocampus is an integral part of Papez or memory circuits, modulation of these structures can be explored as potential therapeutic targets in patients with AD. Fell et al [35] conducted a pilot study to evaluate the effects of low frequency stimulation of EC and hippocampus in 11 patients with temporal lobe epilepsy. They reported a linear correlation of stimulation on correctly remembered words. The best response was seen with in-phase stimulation followed by sham and least with anti-phase stimulation [35]. Another study demonstrated enhancement of spatial memory functions with resetting of theta rhythm on EEG, following in-depth stimulation of EC during learning in seven patients with refractory epilepsy [36]. These studies support the connections between the EC and hippocampus and their role in modulating the memory or learning processes.

\section{Pedunculopontine tegmental nucleus (PPN)}

Stefani et al [37] implanted bilateral PPN DBS in addition to STN DBS in six patients with advanced PD-related dementia. Low frequency stimulation $(25 \mathrm{~Hz})$ of the PPN improved both attention and executive functions. Increased glucose metabolism was noted in the bilateral frontal, prefrontal cortical areas and left ventral striatum following stimulation. Stimulation parameters used in this study were $2.4 \mathrm{~V}, 25 \mathrm{~Hz}$ frequency and $60 \mu$ s pulse width. This study showed that PPN DBS has a potentiating effect on both associative and limbic pathways and can be a potential target for DBS in patients with AD.

\section{Anterior limb of internal capsule/nucleus accumbens (ALIC/NAc)}

Ventral capsule provides a conduit for the white matter tracts originating from the basal ganglia, brain stem and hippocampus and projecting to the frontal lobe. Ventral striatum and nucleus accumbens has also been implicated in motivation and is a part of reward circuitry [38]. Modulation of these pathways has been shown to have beneficial effects in a variety of disorders including obsessive compulsive disorders, depression, obesity, addiction and anxiety disorders [7, 25, 38]. Therefore, modulating these neural circuits might improve the cognitive and behavior functions in patients in AD. The initial results of the study involving bilateral DBS of ALIC/NAc in patients with $\mathrm{AD}$ are promising and yet to be published.

\section{Conclusion}

$\mathrm{AD}$ is a complex disease with significant financial and healthcare burden. The medical therapy for AD is of modest benefit and associated with significant side effects. The use of neuromodulation in the treatment of AD is a relatively newer modality and the most optimal target is yet to be elucidated. DBS of the fornix/hypothalamus has shown promising results in terms of both delaying and reversing the cognitive deterioration in patients with AD. Other targets such as NBM, PPN, ALIC/ NAc and EC have been explored with good initial results. In appropriately selected patients, DBS can potentially delay the cognitive decline, enhance memory functions and can improve the overall quality of life. However, long-term randomized controlled trials are required to validate the efficacy of neuromodulation and to determine the most optimal target for AD.

\section{Disclosures}

The authors have no conflicts of interest or financial disclosures.

\section{Sources of Support}

Nil.

\section{Conflict of Interest}

Nil.

\section{Abbreviations}

DBS: deep brain stimulator; AD: Alzheimer disease; APP: amyloid precursor protein; NBM: nucleus basalis of Meyernet; PPN: pedunculopontine nucleus; ALIC/NAc: anterior limb of internal capsule/nucleus accumbens

\section{References}

1. Shampo MA, Kyle RA, Steensma DP. Alois Alzheimer- 
-Alzheimer disease. Mayo Clin Proc. 2013;88(12):e155.

2. Rocca WA, Petersen RC, Knopman DS, Hebert LE, Evans DA, Hall KS, Gao S, et al. Trends in the incidence and prevalence of Alzheimer's disease, dementia, and cognitive impairment in the United States. Alzheimers Dement. 2011;7(1):80-93.

3. Pereira JL, Downes A, Gorgulho A, Patel V, Malkasian D, De Salles A. Alzheimer's disease: The role for neurosurgery. Surg Neurol Int. 2014;5(Suppl 8):S385-390.

4. Ballard C, Gauthier S, Corbett A, Brayne C, Aarsland D, Jones E. Alzheimer's disease. Lancet. 2011;377(9770):1019-1031.

5. Zibly Z, Shaw A, Harnof S, Sharma M, Graves C, Deogaonkar M, Rezai A. Modulation of mind: therapeutic neuromodulation for cognitive disability. J Clin Neurosci. 2014;21(9):1473-1477.

6. Massoud F, Leger GC. Pharmacological treatment of Alzheimer disease. Can J Psychiatry. 2011;56(10):579-588.

7. Rezai RA, Sharma M. Deep Brain Stimulation (DBS): Current and Emerging Applications. Japanese Journal of Neurosurgery. 2014;23:648-660.

8. Lyketsos CG, Targum SD, Pendergrass JC, Lozano AM. Deep brain stimulation: a novel strategy for treating Alzheimer's disease. Innov Clin Neurosci. 2012;9(11-12):1017.

9. Watt AD, Villemagne VL, Barnham KJ. Metals, membranes, and amyloid-beta oligomers: key pieces in the Alzheimer's disease puzzle? J Alzheimers Dis. 2013;33(Supp1 1):S283-293.

10. Buckner RL, Snyder AZ, Shannon BJ, LaRossa G, Sachs R, Fotenos AF, Sheline YI, et al. Molecular, structural, and functional characterization of Alzheimer's disease: evidence for a relationship between default activity, amyloid, and memory. J Neurosci. 2005;25(34):7709-7717.

11. Sperling RA, Laviolette PS, O'Keefe K, O'Brien J, Rentz DM, Pihlajamaki M, Marshall G, et al. Amyloid deposition is associated with impaired default network function in older persons without dementia. Neuron. 2009;63(2):178-188.

12. Norton S, Matthews FE, Barnes DE, Yaffe K, Brayne C. Potential for primary prevention of Alzheimer's disease: an analysis of population-based data. Lancet Neurol. 2014;13(8):788-794.

13. Arrieta-Cruz I, Pavlides C, Pasinetti GM. Deep Brain Stimulation in Midline Thalamic Region Facilitates Synaptic Transmission and Shortterm Memory in a Mouse Model of Alzheimer's Disease. Transl Neurosci. 2010;1(3):188-194.

14. Hamani C, Stone SS, Garten A, Lozano AM, Winocur G. Memory rescue and enhanced neurogenesis following electrical stimulation of the anterior thalamus in rats treated with corticosterone. Exp Neurol. 2011;232(1):100104.

15. Gao F, Guo Y, Zhang H, Wang S, Wang J, Wu JM, Chen $\mathrm{Z}$, et al. Anterior thalamic nucleus stimulation modulates regional cerebral metabolism: an FDG-MicroPET study in rats. Neurobiol Dis. 2009;34(3):477-483.

16. Miocinovic S, Somayajula S, Chitnis S, Vitek JL. History, applications, and mechanisms of deep brain stimulation.
JAMA Neurol. 2013;70(2):163-171.

17. Robison RA, Taghva A, Liu CY, Apuzzo ML. Surgery of the mind, mood, and conscious state: an idea in evolution. World Neurosurg. 2013;80(3-4):S2-26.

18. Henderson JM. Vagal nerve stimulation versus deep brain stimulation for treatment-resistant depression: show me the data. Clin Neurosurg. 2007;54:88-90.

19. Malone DA, Jr., Dougherty DD, Rezai AR, Carpenter LL, Friehs GM, Eskandar EN, Rauch SL, et al. Deep brain stimulation of the ventral capsule/ventral striatum for treatment-resistant depression. Biol Psychiatry. 2009;65(4):267-275.

20. Pereira EA, Green AL, Nandi D, Aziz TZ. Deep brain stimulation: indications and evidence. Expert Rev Med Devices. 2007;4(5):591-603.

21. Fisher R, Salanova V, Witt T, Worth R, Henry T, Gross R, Oommen $\mathrm{K}$, et al. Electrical stimulation of the anterior nucleus of thalamus for treatment of refractory epilepsy. Epilepsia. 2010;51(5):899-908.

22. Jenkins B, Tepper SJ. Neurostimulation for primary headache disorders: Part 2, review of central neurostimulators for primary headache, overall therapeutic efficacy, safety, cost, patient selection, and future research in headache neuromodulation. Headache. 2011;51(9):1408-1418.

23. Kopell BH, Greenberg B, Rezai AR. Deep brain stimulation for psychiatric disorders. J Clin Neurophysiol. 2004;21(1):51-67.

24. Halpern CH, Wolf JA, Bale TL, Stunkard AJ, Danish SF, Grossman M, Jaggi JL, et al. Deep brain stimulation in the treatment of obesity. J Neurosurg. 2008;109(4):625634.

25. Kuhn J, Lenartz D, Huff W, Lee S, Koulousakis A, Klosterkoetter J, Sturm V. Remission of alcohol dependency following deep brain stimulation of the nucleus accumbens: valuable therapeutic implications? J Neurol Neurosurg Psychiatry. 2007;78(10):1152-1153.

26. Langevin JP, De Salles AA, Kosoyan HP, Krahl SE. Deep brain stimulation of the amygdala alleviates post-traumatic stress disorder symptoms in a rat model. J Psychiatr Res. 2010;44(16):1241-1245.

27. Yamamoto T, Katayama Y. Deep brain stimulation therapy for the vegetative state. Neuropsychol Rehabil. 2005;15(3-4):406-413.

28. Laxton AW, Tang-Wai DF, McAndrews MP, Zumsteg D, Wennberg R, Keren R, Wherrett J, et al. A phase I trial of deep brain stimulation of memory circuits in Alzheimer's disease. Ann Neurol. 2010;68(4):521-534.

29. Hamani C, McAndrews MP, Cohn M, Oh M, Zumsteg D, Shapiro CM, Wennberg RA, et al. Memory enhancement induced by hypothalamic/fornix deep brain stimulation. Ann Neurol. 2008;63(1):119-123.

30. Smith GS, Laxton AW, Tang-Wai DF, McAndrews MP, Diaconescu AO, Workman CI, Lozano AM. Increased cerebral metabolism after 1 year of deep brain stimulation in Alzheimer disease. Arch Neurol. 2012;69(9):11411148.

31. Fontaine D, Deudon A, Lemaire JJ, Razzouk M, Viau P, Darcourt J, Robert P. Symptomatic treatment of memory decline in Alzheimer's disease by deep brain stimulation: 
a feasibility study. J Alzheimers Dis. 2013;34(1):315323.

32. Koubeissi MZ, Kahriman E, Syed TU, Miller J, Durand DM. Low-frequency electrical stimulation of a fiber tract in temporal lobe epilepsy. Ann Neurol. 2013;74(2):223231.

33. Boix-Trelis N, Vale-Martinez A, Guillazo-Blanch G, Costa-Miserachs D, Marti-Nicolovius M. Effects of nucleus basalis magnocellularis stimulation on a socially transmitted food preference and c-Fos expression. Learn Mem. 2006;13(6):783-793.

34. Freund HJ, Kuhn J, Lenartz D, Mai JK, Schnell T, Klosterkoetter J, Sturm V. Cognitive functions in a patient with Parkinson-dementia syndrome undergoing deep brain stimulation. Arch Neurol. 2009;66(6):781-785.

35. Fell J, Staresina BP, Do Lam AT, Widman G, Helmstae- dter C, Elger CE, Axmacher N. Memory modulation by weak synchronous deep brain stimulation: a pilot study. Brain Stimul. 2013;6(3):270-273.

36. Suthana N, Haneef Z, Stern J, Mukamel R, Behnke E, Knowlton B, Fried I. Memory enhancement and deepbrain stimulation of the entorhinal area. $\mathrm{N}$ Engl J Med. 2012;366(6):502-510.

37. Stefani A, Pierantozzi M, Ceravolo R, Brusa L, Galati S, Stanzione P. Deep brain stimulation of pedunculopontine tegmental nucleus (PPTg) promotes cognitive and metabolic changes: a target-specific effect or response to a low-frequency pattern of stimulation? Clin EEG Neurosci. 2010;41(2):82-86.

38. Bichot NP, Heard MT, Desimone R. Stimulation of the nucleus accumbens as behavioral reward in awake behaving monkeys. J Neurosci Methods. 2011;199(2):265-272. 